Pamela B. Davis (iD) ${ }^{凶}$, Emma A. Meagher ${ }^{2}$ Claire Pomeroy ${ }^{3}$, William L. Lowe Jr(B) ${ }^{4}$, Arthur H. Rubenstein ${ }^{2}$, Joy Y. Wu (D) ${ }^{5}$, Anne B. Curtis ${ }^{6}$ and Rebecca D. Jackson ${ }^{7}$ ${ }^{1}$ Center for Community Health Integration, Case Western Reserve University School of Medicine, Cleveland, OH, USA. ${ }^{2}$ Department of Medicine, Raymond and Ruth Perelman School of Medicine, University of Pennsylvania, Philadelphia, PA, USA. ${ }^{3}$ Albert and Mary Lasker Foundation, New York, NY, USA. ${ }^{4}$ Department of Medicine, Northwestern University Feinberg School of Medicine, Chicago, IL, USA. ${ }^{5}$ Department of Medicine, Stanford University School of Medicine, Stanford, CA, USA. ${ }^{6}$ Department of Medicine, Jacobs School of Medicine and Biomedical Sciences, University at Buffalo, Buffalo, NY, USA. 'Department of Medicine, The Ohio State University, Columbus, OH, USA.

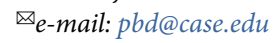

Published online: 17 February 2022 https://doi.org/10.1038/s41591-022-01692-8

\section{References}

1. Myers, K. R. et al. Nat. Hum. Behav. 4, 880-883 (2020).

2. Ewing-Nelson, C. National Women's Law Center https://nwlc.org/ wp-content/uploads/2021/01/December-Jobs-Day.pdf (January 2021)
3. Mandavilli, A. The New York Times https://www.nytimes.com/ 2021/04/13/health/women-stem-pandemic.html (accessed 6 December 2021).

4. Matulevicius, S. A. et al. JAMA Netw. Open 4, e2113539 (2021).

5. Wehner, M. R., Li, Y. \& Nead, K. T. I. JAMA Netw. Open 3 , e2020335 (2020).

6. Pinho-Gomes, A. C. et al. BMJ Glob. Health 5, e002922 (2020).

7. Wu, J. The Clayman Institute for Gender Research https://gender. stanford.edu/news-publications/gender-news/pandemicmagnifies-gender-inequities-provides-opportunities-long-range (19 July 2021).

8. Lauer, M. NIH Extramural Nexus https://nexus.od.nih.gov/all/ 2021/06/01/an-updated-look-at-applications-submittedduring-the-pandemic/ (1 June 2021).

9. Antecol, H. et al. Am. Econ. Rev. 108, 2420-2441 (2018). 10. Richter, K. P. et al. N. Engl. J. Med. 383, 2148-2157 (2020).

11. U.S. Department of Labor Women's Bureau. https://www.dol.gov/ agencies/wb/news/newsletter/march2021 (March 2021).

12. Lo Sasso, A. T. et al. Health Aff. 39, 256-263 (2020).

13. Hechtman, L. A. et al. Proc. Natl Acad. Sci. USA 115, 7943-7948 (2018).

14. Oliveira, D. F. M. et al. J. Am. Med. Assoc. 321, 898-900 (2019).

15. Kibbe, M. R. JAMA Surg. 195, 803-804 (2020).

16. Burstin, H. R. \& Arora, V. M. JAMA Netw. Open 4, e 2114787 (2021).

17. Chaterjee, P. \& Werner, R. M. JAMA Netw. Open 4, e2114509 (2021).

18. Jagsi, R. et al. Arch. Intern. Med. 167, 343-345 (2007).

19. Jones, R. D. et al. Acad. Med. 94, 1746-1756 (2019).

20. Jagsi, R. et al. Ann. Intern. Med. 169, 128-130 (2018).

21. US National Institutes of Health. https://grants.nih.gov/ grants/guide/notice-files/NOT-OD-20-055.html (accessed 6 December 21).

22. US National Institutes of Health. https:/grants.nih.gov/grants/ guide/notice-files/NOT-OD-20-054.html (accessed 6 December 21)
23. Fuentes-Afflick, E. \& Higginbotham, E. N. Engl. J. Med. 384, 2265-2267 (2021).

24. National Academies of Sciences, Engineering, and Medicine. The Impact of COVID-19 on the Careers of Women in Academic Sciences, Engineering, and Medicine (eds. Higginbotham, E. \& Dahlberg, M.L.) (The National Academies Press, 2021); https://doi.org/10.17226/26061

25. Clinical Research Forum. https://www.clinicalresearchforum.org/ page/impactofthepandemic (2022).

\section{Acknowledgements}

We gratefully acknowledge the Clinical Research Forum Board of Directors for constituting the Academic

Advancement Committee, whose members became the authors of this paper, for helpful discussion of the data and the recommendations, and for their support of women in academic medicine. Other than authors of this paper, the members of the Clinical Research Forum Board are

H. Selker, H. Pardes, R. Califf, B. Coller, M. Fava, H. Greenberg, R. Kaushal, A. Reece, A. Shenkhar and D. Ford.

\section{Author contributions}

P.B.D. wrote the manuscript and integrated edits from other authors. Important discussion and extensive editing was provided by all authors.

\section{Competing interests}

P.B.D., E.A.M., W.L.L., A.H.R., A.B.C. and R.D.J. serve on the Board of Directors of the Clinical Research Forum. R.D.J. has received a grant from the Doris Duke Charitable Foundation. P.B.D., C.P. and A.H.R. are members of the Board of Directors of the Center for Women in Academic Medicine and Science.

\title{
The hospital-at-home presents novel liabilities for physicians, hospitals, caregivers, and patients
}

Healthcare is increasingly provided in a patient's home, with potential cost savings and clinical improvements. But the hospital-at-home also raises unique liability issues not only for physicians and hospitals but also for caregivers and patients.

\section{David A. Simon, I. Glenn Cohen, Celynne Balatbat and Anaeze C. Offodile II}

T he COVID-19 pandemic has spurred hospitals to increasingly implement remote care models that replace in-patient care - from checkups to diagnosis to treatment - with services delivered in the patient's home, known as the 'hospital-at-home' (HaH) (Box 1). Such patients typically move through the $\mathrm{HaH}$ in the same way that a patient moves through a traditional hospital: admission, monitoring, intervention, and discharge. But there are differences. Care occurs in the patient's home, rather than in the hospital, which requires that the $\mathrm{HaH}$ providers use physicians, nurses, technicians, and caregivers in different ways from their use in the traditional hospital setting, such as telehealth or house visits. The $\mathrm{HaH}$ has potentially substantial economic and health benefits and has been associated with reduced risk of infections, increased mobility for patients, cost savings, and improved clinical outcomes ${ }^{1,2}$. Despite these potential benefits, the $\mathrm{HaH}$ poses several pressing legal and ethical challenges, including legal liability, that must be resolved for its safe, ethical, and effective implementation.

A clinical vignette (Fig. 1) can be used to demonstrate and map the unique and complex liability issues likely to arise in the HaH setting, including liability for physicians, hospitals, and third-party service providers ${ }^{3}$. Some potential liability, such as liability for admission decisions or delayed response times, exists in the hospital setting but applies to the $\mathrm{HaH}$ in a unique way. Other potential liability, such as liability arising from dangerous conditions at the patient's home, is unique to the $\mathrm{HaH}$ setting (Table 1).

\section{Physician liability}

Under US tort law, when caring for patients, physicians must provide a certain standard of care: they must, depending on the state in which they practice, act either according to the "generally recognized and accepted practices in their profession" or as a "reasonable physician under similar circumstances" would act ${ }^{4-6}$. Although 


\section{Box 1 | Defining the $\mathrm{HaH}$}

An $\mathrm{HaH}$ is defined as a care-delivery paradigm characterized by the following:

A pre-specified geographic catchment area for eligible patients that is delineated largely by travel time

An acuity and intensity of medical care needs that would otherwise warrant admission to an inpatient facility

An inability of community-based home health services to appropriately coordinate and deliver the services needed to sufficiently address the severity of illness

Note that this definition of $\mathrm{HaH}$ includes both a standard hospital substitutive model of care, as well as alternative care-delivery frameworks that go beyond simply replacing the in-patient acute care experience, including rehabilitation at home and hospice at home ${ }^{24}$.

standards of care apply equally to $\mathrm{HaH}$ care (physicians and hospitals have duties to exercise reasonable care in admitting, monitoring, and treating patients), courts may apply them differently in the $\mathrm{HaH}$ context.

Consider a physician's decision to admit a patient. In the traditional setting, the physician evaluates the patient's symptoms, the capacities of the hospital, and level of care required. In the $\mathrm{HaH}$ context, because the care setting is at home, the physician must also account for the readiness of the home environment, caregiver availability, and other social determinants of health or factors not relevant to the hospital setting.

In the clinical vignette here (Fig. 1), it is unclear whether the physician in the emergency department properly admitted the patient to the $\mathrm{HaH}$ program, given that 24-hour nursing and ancillary services (such as imaging, labs, and intravenous medications) are not readily available there, so that an acute change in a patient's physical condition cannot be closely monitored and mitigated. Unlike inpatient settings, $\mathrm{HaH}$ check-ins are scheduled and do not involve specialized staff, which leads to a much lower likelihood that an abrupt change in health status will be detected, relative to the likelihood in a traditional hospital. The $\mathrm{HaH}$ may also involve remote monitoring devices that record and transmit patient-generated health information (such as vital signs, sleep quality, and symptom burden) to hospital staff. If sensor data trigger an urgent in-person evaluation, the $\mathrm{HaH}$ response protocols will also need to account for additional time (i.e., transit duration) that in-patient settings can avoid because staff operate in close physical proximity to the patient.

The liability system needs to adapt to the $\mathrm{HaH}$. Telemedicine provides a possible analogy. Some US states have enacted laws that apply the same standard of care to in-person and telemedicine physician encounters, whereas at least one state (Hawaii) has specified a separate standard for services rendered in a telemedicine encounter? To clarify the law in this area, lawmakers should consider proactively legislating what standard of care applies in the $\mathrm{HaH}$ context.

Because physicians will need to determine when and whether to send a response team, the $\mathrm{HaH}$ also affects the physician's responsibility to check in on the patient. The physician has a legal duty to monitor the patient, so a care team might be required to check in more frequently in the $\mathrm{HaH}$ setting than in the hospital setting. Furthermore, determining whether the care team adequately met its duty to respond to a care episode in the $\mathrm{HaH}$ might depend on factors outside human control, such as an extraordinary and unforeseeable event (force majeure) or traffic patterns. It is unclear if the physician is liable when a response team is deployed in a timely fashion but its arrival is delayed for reasons, such as unexpected traffic or inclement weather, that would not have occurred in the traditional setting. It is also not clear if the standard of care for response time should be judged according to what would have been appropriate in the hospital or what is reasonable given the travel distance from the hospital to the patient. Driving 40 blocks to a patient in a congested city is quite different from taking an elevator down three floors.

The clinical vignette here (Fig. 1) shows how an admission decision to the $\mathrm{HaH}$ encompasses additional complex non-medical factors that extend beyond clinical judgment on how best to treat a patient, such as the inability to administer critical medications to a patient in the $\mathrm{HaH}$ setting. Health systems implementing the $\mathrm{HaH}$ will need to develop criteria for admission and care escalation (such as urgent transfer back to the facility setting) that incorporate non-medical factors, such as traffic patterns or feasibility of medication administration.

\section{Hospital and third-party liability}

Care decisions fall mainly on physicians, but liability for these decisions can extend to hospitals as well. The hospital is liable for the acts of its employee-physicians acting within the scope of employment. The hospital is also liable for the acts of independent contractors whom the patient reasonably believes are employees or who act with the authority of the hospital ${ }^{8}$. For example, if the patient reasonably believed that the physician in the emergency department was an agent of the hospital, even if the physician was not an employee, the hospital could still be liable for the physician's actions.

Third-party service providers can be liable for acts committed in the course of providing services related to the $\mathrm{HaH}$, such as a home health attendant who is advertised as 'part of the care team' but is not employed by the hospital. If the home health attendant fails to administer medication, or negligently mis-records information about the patient, and the patient is harmed as a result, the hospital could face liability for the attendant's actions. Likewise, a mobile $\mathrm{X}$-ray technician who negligently captures images that result in an erroneous treatment decision could render the hospital liable for the technician's negligence if the patient reasonably believed that the technician worked for the hospital'.

Although many hospitals contract with third-party service providers, the $\mathrm{HaH}$ context involves an intricate web of contractors whom the patient may, justifiably, come to view as a single care team. If an $\mathrm{HaH}$ program uses third-party providers to administer services without sufficiently informing the patient that they are not hospital employees, the hospital may become liable for the acts of these third parties. Some states have enacted statutes that immunize (i.e., exempt from liability) the negligent (but sometimes not the grossly negligent or reckless) conduct of particular third-party contractors such as emergency medical technicians, but these laws often apply only to emergency situations ${ }^{10}$. To reduce legal ambiguity, $\mathrm{HaH}$ programs should carefully inform patients which contractors are affiliated with the hospital and which are not. This should include referring to third-party service providers by their corporate or assumed names and an explanation that specific service providers are not employees or agents of the hospital. Under existing case law, however, it is not clear that even these kinds of steps will go far enough to prevent liability flowing back to the $\mathrm{HaH}$ program. Perhaps this is as it should be, but it produces yet another complication in designing $\mathrm{HaH}$ programs.

Hospitals can also be liable for their corporate acts, such as failing to act reasonably in reviewing and supervising the patient's care, granting admission privileges to a physician, and setting policies 


\begin{tabular}{|c|c|c|c|}
\hline $\begin{array}{l}\text { Emergency department } \\
\text { assessment }\end{array}$ & HaH admission & HaH care delivery & Care escalation \\
\hline $\begin{array}{l}\text { - Emergency department } \\
\text { evaluation and } \\
\text { diagnosis of pneumonia } \\
\text { - Confirmation of } \mathrm{HaH} \\
\text { eligibility criteria } \\
\text { - Caregiver education on } \\
\text { HaH, focused on } \\
\text { providing detailed } \\
\text { description of HaH } \\
\text { program }\end{array}$ & $\begin{array}{l}\text { - Admission orders } \\
\text { - Documentation } \\
\text { - Transfer to the home } \\
\text { with remote sensors, } \\
\text { home oxygen, daily } \\
\text { check-ins via video } \\
\text { visits, parenteral } \\
\text { infusion equipment for } \\
\text { medications, IV fluids, } \\
\text { etc. }\end{array}$ & $\begin{array}{l}\text { - On day } 2 \text {, patient noted } \\
\text { to be somnolent by } \\
\text { caregiver } \\
\text { - Scheduled vitals } \\
\text { check-in took place } 3 \\
\text { hours later and results } \\
\text { were found to be } \\
\text { aberrant } \\
\text { - Emergent response } \\
\text { activated }\end{array}$ & $\begin{array}{l}\text { Within } 90 \text { minutes, } \\
\text { in-person team arrived } \\
\text { for initial stabilization at } \\
\text { home. Emergent } \\
\text { transfer to hospital } \\
\text { - Admitted to ICU for } \\
\text { definitive stabilization, } \\
\text { on ventilator, and } \\
\text { treatment }\end{array}$ \\
\hline
\end{tabular}

Fig. 1 | Clinical vignette of an $\mathrm{HaH}$ patient. The patient is a 61-year-old male, otherwise healthy, who recently initiated outpatient-based chemotherapy for a locally advanced renal cancer. The patient developed a non-productive cough and shortness of breath after a walk. The patient's wife and primary caregiver immediately took the patient to the nearest emergency department, where the patient began to develop a fever. IV, intravenous; ICU, intensive care unit.

\section{Table 1 | Potential liabilities in the $\mathrm{HaH}$}

\begin{tabular}{ll} 
Actor & Potential liability \\
Physician & $\begin{array}{l}\text { Admission and discharge decisions; failure to adequately monitor; failure to } \\
\text { warn; failure to respond in a timely fashion; delays in treatment }\end{array}$ \\
\hline Hospital & $\begin{array}{l}\text { Acts of physicians, staff, and contractors; failure to develop or implement } \\
\text { reasonable policies and best practice around care delivery in the HaH; failure } \\
\text { to educate or inform caregiver }\end{array}$ \\
\hline Third-party provider & $\begin{array}{l}\text { Delay in response time; ineffective or inappropriate care delivery } \\
\text { Caregiver }\end{array}$ \\
\hline Patient & $\begin{array}{l}\text { Insufficient patient monitoring; ineffective administration of medication } \\
\text { contractors by the patient, or by conditions or individuals on the patient's } \\
\text { property }\end{array}$ \\
\hline
\end{tabular}

and practice guidelines that hospital staff must follow ${ }^{11-14}$. Because $\mathrm{HaH}$ programs are still new and evolving, there are a variety of programmatic issues that could create liability exposure, including staffing, admission and discharge protocols, and care monitoring. This may also be a fertile area for the development of comprehensive clinical-practice guidelines.

\section{Caregiver liability}

A fairly unique feature of $\mathrm{HaH}$ programs is that the caregivers such as family, friends, or paid aides frequently participate in patient management. Generally, caregivers owe a duty of care toward those for whom they care $^{15}$. These people, therefore, may also face liability for their role in caring for the patient.

In the clinical vignette here (Fig. 1), the patient's wife, acting as caregiver, could have a duty to reasonably monitor her husband. In a lawsuit by the patient or his estate, a hospital could defend itself by arguing that the patient's wife breached her duty by failing to contact the hospital in a timely fashion when the patient was difficult to arouse. If under the circumstances a reasonable person would have contacted the hospital, the caregiver could be partially or fully liable for the harm, which would potentially limit the hospital's exposure. Because caregivers are often unpaid or are hired by the family, they may be considered volunteers, independent contractors, or employees of the family. Without a formal employment or other contractual relationship with the $\mathrm{HaH}$ program, the hospital would not, absent special circumstances, be liable for the acts or inaction of caregivers with whom the patient or the patient's family contracts. Although this clinical vignette does not involve any other family members, anyone who provides assistive care could potentially be swept up in a lawsuit.

Hospitals can proactively address these concerns by ensuring that their $\mathrm{HaH}$ best practices and protocols are robust with respect to caregiver education and caregiver respite. Hospitals should also ensure that the caregiver consent process is comprehensive and clearly articulates the roles and responsibilities of caregivers, distinct from those of the $\mathrm{HaH}$ program staff, using culturally and linguistically appropriate language. Hospitals must be careful not to exercise too much control over the caregiver, or to give the impression the caregiver has authority to act on behalf of the hospital, lest they open themselves to the kinds of liability discussed above.

\section{Device-manufacturer liability}

The HaH will require that staff and physicians synchronously and asynchronously monitor patients. If monitoring or other devices malfunction, are defective, or are improperly used, device manufacturers could face liability. Hospitals should pay careful attention to any user agreements or contracts they sign with device manufacturers that could shift or limit liability of device manufacturers, potentially at the expense of hospitals and physicians. Hospitals should also consider developing protocols that readily detect and account for device failure. Ideally, these should include supply-chain redundancy and automated remote checks, as well as periodic efficacy and safety assessments by dedicated personnel to ensure device integrity, although such measures may raise costs for the $\mathrm{HaH}$.

\section{Intentional and unintentional harms}

There are other issues that arise uniquely in the $\mathrm{HaH}$ setting without a ready analogue in the traditional hospital context. For example, hospitals are able to exert considerable control over the number and types of interactions an inpatient has. In the $\mathrm{HaH}$ setting, hospitals have far less control, although they may still be liable when a third party with whom they have a relationship, such as a contractor or emergency medical technician, intentionally harms the patient ${ }^{16,17}$. Although this liability may not extend to unforeseeable criminal or tortious acts by third parties, hospitals may, in some cases, have a duty to report and act upon, if not monitor, abuse that they witness because of their special relationship with the patient $^{18-20}$.

Hospitals should carefully screen contractors who enter a patient's home, provide to the patient the names and 
photographs of contractors, and inform the patient if a contractor is abruptly terminated or could otherwise pose a risk to the patient. However, hospitals must also be careful not to disclose more information than is necessary to inform the patient of a potential risk. Disclosing too much information, or disclosing certain types of information, may open hospitals to additional liability.

Risks can also arise for $\mathrm{HaH}$ personnel and third-party service providers from conditions or actors at the patient's home, including risk from the patient themself. These harms may occur accidentally from dangerous conditions at the home, such as a loaded gun that discharges and injures a caregiver, an exposed live wire that electrocutes a nurse while they are preparing a bath, or a deteriorating wall or ceiling that collapses on a third-party service provider ${ }^{21}$. Intentional harms may be committed by the patient or third parties, such as physical harm to the caregiver (assault and/or battery $)^{22,23}$.

\section{Risk of discrimination}

It is unclear what duty, if any, an $\mathrm{HaH}$ program has to investigate whether dangerous persons or conditions exist at the patient's home and whether these risks should factor into the hospital's admission criteria. If an $\mathrm{HaH}$ program knows or should know of a dangerous, or potentially dangerous, condition at the patient's home, it may have legal obligations to notify its employees or third-party service providers of that condition or protect them from it. Liability, if imposed in cases such as these, may prompt the $\mathrm{HaH}$ to change its admission criteria, which may then reduce inclusion of the populations most likely to need $\mathrm{HaH}$ care.

These challenges are particularly pronounced for $\mathrm{HaH}$ implementation in areas with economic deprivation or high crime rates. Some hospitals may be reluctant to use the $\mathrm{HaH}$ in these settings, because of concern for the actual or perceived safety of their staff. If an $\mathrm{HaH}$ program considers such conditions in deciding whom to admit, it may face claims of discrimination, or legal challenges. The $\mathrm{HaH}$ administrators confronting such risks face the unenviable burden of balancing the risks to their employees and business against the benefits, both to the patient and the hospital, of delivering care to those who need it most. Hospitals using artificial intelligence systems to assess patient suitability for $\mathrm{HaH}$ admission may also face additional ethical risks, in that these systems can often embed preexisting biases into decision-making. The best approach would be to leverage a diverse and multi-disciplinary team in the creation of the $\mathrm{HaH}$ model, and establish a governance structure that includes the patient community, combined with stratified public reporting of $\mathrm{HaH}$ enrollment by ethnicity, age, sex, and income.

\section{Guidelines and training}

$\mathrm{HaH}$ programs hold great promise but also present novel liability concerns. Decisions about admission to the $\mathrm{HaH}$ invite a unique set of considerations that go beyond the appropriateness of hospital-level acuity to include home safety, logistics, technological limitations, and cultural considerations, as well as potential liability for caregivers and for patients themselves. Professional societies and hospital associations should proactively establish practice guidelines and training curricula for the $\mathrm{HaH}$ to enhance its implementation and scalability, and to reduce liability risks.

\section{David A. Simon'1 I. Glenn Cohen (D) 1,2四, Celynne Balatbat ${ }^{3}$ and Anaeze C. Offodile II ${ }^{4}$ ${ }^{1}$ Petrie-Flom Center for Health Law Policy, Biotechnology, and Bioethics, Harvard Law School, Cambridge, MA, USA. ${ }^{2}$ Harvard Law School, Cambridge, MA, USA. ${ }^{3}$ National Academy of Medicine, Washington, DC, USA. ${ }^{4}$ Department of Plastic Surgery, Division of Surgery, The University of Texas MD Anderson Cancer Center, Houston, TX, USA. \\ 凶e-mail: igcohen@law.harvard.edu}

Published online: 17 February 2022

https://doi.org/10.1038/s41591-022-01697-3

References

1. American Hospital Association. https://www. aha.org/system/files/media/file/2020/12/

issue-brief-creating-value-by-bringing-hospital-care-home_0.pdf (2020).

2. American Hospital Association. https://www.aha.org/ case-studies/2018-09-12-brigham-and-womens-hospital-boston (accessed 4 October 2021)

3. Gerke, S. et al. J. Am. Med. Assoc. 323, 601-602 (2020).

4. Peters, P. G. Wash. Lee Law Rev 57, 163-205 (2000).

5. Doe v. Am. Red Cross Blood Serv., 377 S.E.2d 323, 326 (S.C. 1989).

6. Weiss v. Pratt, 53 So. 3d 395, 401 (Fla. Dist. Ct. App. 2011).

7. Haw. Rev. Stat. $\$ 453-1.3(\mathrm{c})$.

8. Cefaratti v. Aranow, 141 A.3d 752, 759 (Conn. 2016).

9. Pamperin v. Trinity Mem'l Hosp., 423 N.W.2d 848, 856 (Wis. 1988).

10. Cooper v. Rodriguez, 118 A.3d 829, 832 (Md. 2015).

11. Darling v. Charleston Cmty. Mem'l Hosp., 211 N.E.2d 253, 256 (Ill. 1965).

12. Gilbert v. Sycamore Mun. Hosp., 518, 622 N.E.2d 788, 792 (Ill. 1993).

13. Pedroza v. Bryant, 677 P.2d 166 (Wash. 1984).

14. Cefaratti v. Aranow, 141 A.3d 752, 766 (Conn. 2016).

15. Arkansas Rev. Stat. $\$ \$ 46-456$.

16. Young v. Huntsville Hosp., 595 So. 2d 1386 (Ala. 1992)

17. Jankee v. Clark Cty., 612 N.W.2d 297, 321 (Wis. 2000).

18. 23 Pa.C.S. $\$ \$ 6311(\mathrm{a}), 6318$.

19. Jankee, 612 N.W.2d at 321.

20. McSwane v. Bloomington Hosp. \& Healthcare Sys., 916 N.E.2d 906, 910 (Ind. 2009).

21. Hernandez v. Jensen, Cal. Rptr. 3d 281, 286 (Cal. App. 2d Dist. 2021).

22. Sanders v. Alger, 394 P.3d 1083, 1086 (Ariz. 2017) (duty generally)

23. White v. Muniz, 999 P.2d 814 (Colo. 2000) (intentional torts).

24. Lee, B. \& Montalto, M. J. Am. Geriatr. Soc. 52, 2141

(2004).

\section{Acknowledgements}

D.A.S. and I.G.C. receive funding support from the Moore Foundation (grant 9974). A.C.O. receives funding support from the Rising Tide Foundation for Clinical Cancer Research (grant CCR-21-500).

\section{Author contributions}

D.A.S. led the composition of the initial manuscript. All authors contributed to the writing, editing and revising of the manuscript.

\section{Competing interests}

I.G.C. serves as a bioethics consultant for Otsuka on the Abilify MyCite product, is a member of the Illumina and Bayer ethics advisory boards, and serves as an ethics consultant for DawnLight. 